\title{
A Virtual Reality Teaching Simulation for Exercise During Pregnancy
}

\author{
https://doi.org/10.3991/ijet.v14i01.8944
}

Abas Setiawan ${ }^{(凶)}$, Faik Agiwahyuanto, Pramudi Arsiwi

Dian Nuswantoro University, Semarang, Jawa Tengah, Indonesia abas.setiawan@dsn.dinus.ac.id

\begin{abstract}
One of the poor indicators of the development and health status of a country is high maternal mortality. The world has seen that one pregnant woman in every two minutes dies because of complication with serious or longlasting consequences. During pregnancy, pregnant women tend to reduce physical activity due to increased sensitivity factors. It will lead to decrease the elasticity of muscles and joints. The way to improve the elasticity is doing exercise during pregnancy. But, there are still many pregnant women who are less interested in doing exercise during pregnancy, due to high loaded in working day and dense schedule of daily activities in her career or as a housewife. Some women assume that by attending pregnancy exercise course in hospitals or health care centers is time-consuming and too formal because they have to follow the prenatal personal trainer schedule. The technology that allows helping pregnant women in exercise during pregnancy is virtual reality. In this study, the development of virtual reality application for exercise during pregnancy adapted from the methodology to determine when to use virtual reality in education and training combined with the Immersive Virtual Environment (IVE) questionnaire. The average results of overall components in the IVE questionnaire is 4.26 of 5-point scales that indicates the virtual reality application for exercise during pregnancy is feasible to use by pregnant woman.
\end{abstract}

Keywords - maternal mortality, exercise, pregnant woman, virtual reality, IVE

\section{Introduction}

High maternal mortality is a poor indicator of the development and health status of a country. Maternal mortality according to World Health Organization (WHO) reviewed by a woman while pregnant within 42 days of pregnancy termination. High maternal mortality is worsened by risky pregnancy, childbirth process which is handled by incompetent health care, and accidental or incidental cause during pregnancy. Indicators of woman's health during pregnancy assessed with maternal health programs. Maternal health programs are very concerned about health improvement services in accessibility and quality.

According to the United Nations Population Fund, about one pregnant woman in every two minutes who dies because of complication with serious or long-lasting 
consequences [1]. During pregnancy, pregnant women may feel more sensitive to her surroundings. This condition could make pregnant women to reduce physical activity so that the muscles and joints become inelastic and weak. One way that the muscles and joints of pregnant women do not become elastic and weak is doing exercise during pregnancy. Besides that, exercise during pregnancy guides the pregnant woman to get physiological maturation when faced with childbirth process. Doing pregnancy exercise is almost similar to the process of childbirth itself. Some of the benefits of this exercise during pregnancy are reducing anxiety during childbirth process, better digestion, improving appetite, sleeping well, improving the mood of pregnant women [2], and reducing excess weight during pregnancy [3], [4].

In recent years, there are still many pregnant women who are less interested in doing exercise during pregnancy, due to various reasons. The most dominant factor is too busy and dense schedule of daily activities in her career or as a housewife. Some women assume that by attending pregnancy exercise course in hospitals or health care centers is time-consuming and too formal because they have to follow the prenatal personal trainer schedule. It needs a technology that can motivate and help pregnant women to do exercise during pregnancy; one of them is virtual reality.

Virtual reality allows users to be in an artificial environment with experience gained from sight and sound through sensors connected to a computer or a smartphone. Virtual reality can increase the awareness of the situation, clarity, interactivity, and kinesthetic of the centralized experience. Virtual reality provided a virtual world as a visualization. The virtual world affects how immersive the user with the virtual environment [5]. The high immersive level of virtual world makes user attention more focused on what objects seen in the virtual world.

In this study, the development of virtual reality application for exercise during pregnancy adapted from the methodology to determine when to use virtual reality in education and training [6] combined with the user experience evaluation method of Immersive Virtual Environment questionnaire [7]. Immersive Virtual Environment is a computer-based intelligent system interaction that features a three-dimensional virtual world. The IVE questionnaire is designed to be able to measure user experience residing in an immersive virtual environment.

\section{Literature Review}

\subsection{Virtual reality for education}

A conventional type of education uses instructional aids in teaching courses, such as textbooks, lecture notes, simulation, and discussion. Nowadays, educators and trainers may use ordinary computer software like slide-presentation, educational website, blog, film, and specific education software [8]. However, there are some courses which it needs more interactive instructional aids. Virtual reality can be used to teach or to make any simulation with more interactive and efficient ways. Pantelidis describe the reasons to use VR in education and training course and a methodology to determine when to use virtual reality [6]. 
Virtual reality has capabilities to visualize with great details of visual representation. The visual representation of VR can motivate the students to observe the object materials or events. Virtual reality used when training is difficult or dangerous, simulation needed, required models, and information visualization needed. There is a good methodology to determine when to use virtual reality proposed by Pantelidis [6], but there is an undetailed explanation about the evaluation technique.

\subsection{Virtual Reality for health training simulation}

High enthusiasm on developing VR applications make the VR application possible to use for training-based education in the field of health and medical training. Instead of using conventional training system, VR can transfer information more accurately through simulation. The simulation scenarios in a virtual environment can increase the level of knowledge abstraction of the user while recognizing the problem [9]. Yong et al investigate the effect of VR training in unilateral spatial neglect of stroke patients [10]. After some experiment of VR training conducted on patients, the study suggests the VR training can use as an alternative way of therapeutic technique in unilateral spatial neglect of stroke patients. Several VR application for medical surgery also presented for medical training such as spine surgery training simulator [11] and medical surgery [12].

Chen et al use VR to train patients with chronic neck pain and kinesiophobia [13]. By altering visual feedback, VR is useful to increase the range of neck motion during the training. Microsoft Kinect device was used to take the feedback to enhance the visual perception of the user. The feedback appears in user's perspective as a visual display then the user sees and matches his motion during rehabilitation training in a virtual environment.

The application of VR training can also use to increase physical activities of institutionalized older people [14]. The lack of physical activity of older people in nursing homes can decrease the cognitive functioning. The VR application simulation called PhysEx (Physical Exercise with exergames) proposed to reduce the risk of lacking physical activity and increase the cognitive function.

\subsection{Virtual reality evaluation}

A lot of research has been conducted to measure the user experience in the immersive virtual environment (IVE). Katy Tcha-Tokey et al proposed a questionnaire to measure the VR experience called IVE questionnaire [7]. The IVE questionnaire consists of several components, such as presence, engagement, immersion, flow, usability, skill, emotion, experience consequence, judgment, and technology adoption. Presence is defined as the subjective experience of a person while in the virtual environment, even though the person is physically in the real environment [15]. Engagement is the relationship between people with activities consisting of behavioral, emotional, and cognitive forms. Presence and Engagement can be measured using Presence Questionnaire (PQ). 
Immersion is an illusion, in other words, the virtual environment replaces the user's sensory stimulus with a virtual sensory stimulus. This component can be measured using Immersive Tendency Questionnaire (ITQ) [15]. The flow described as a psychological state of the user feel about the sense of control, joy, and fun when making any interaction in a virtual environment. The flow measured by Flow4D16 questionnaire [16].

Usability is a component that demonstrates the ease of learning (learning) and ease of use (related to efficiency, effectiveness, and satisfaction) of virtual environments. Usability can be measured using System Usability Scale (SUS) [17]. Skill demonstrates user knowledge in mastering activities performed in a virtual environment and can be measured using the Computer Self-Efficacy (CSE) questionnaire [18]. Emotion shows the user's feelings in a virtual environment, which includes pleasure, satisfaction, frustration, anxiety, and so on. These emotion components can be measured using the Achievement Emotions Questionnaire (AEQ) [19].

Experience consequence is a perceived symptom of the user after being in a virtual environment, such as stress, headache, and so on. This component can be measured using the Simulator Sickness Questionnaire (SSQ) designed by Kennedy in 1993 [20]. Technology adoption defined the actions and decisions are taken by the user while responding to every event in a virtual environment. The component measured using the Unified Technology Acceptance and Use of Technology (UTAUT) questionnaire created by Venkatesh in 2003 [21].

\section{Research Methodology}

There are ten steps in the Pantelidis's methodology to determine when to use VR [6]. But, there is no detailed explanation of how to evaluate the user experience gained after using the VR application. We proposed methodology with adding an evaluation step with the IVE questionnaire [7].

In this case, there are additional sub-steps before implementation and after implementation the VR for pregnancy exercise. The sub-step is doing vital sign check-up. It is important to check pregnant women who have a medically unstable condition before using VR. Fig. 1 depicts a complete research methodology adopted by Pantelidis's methodology.

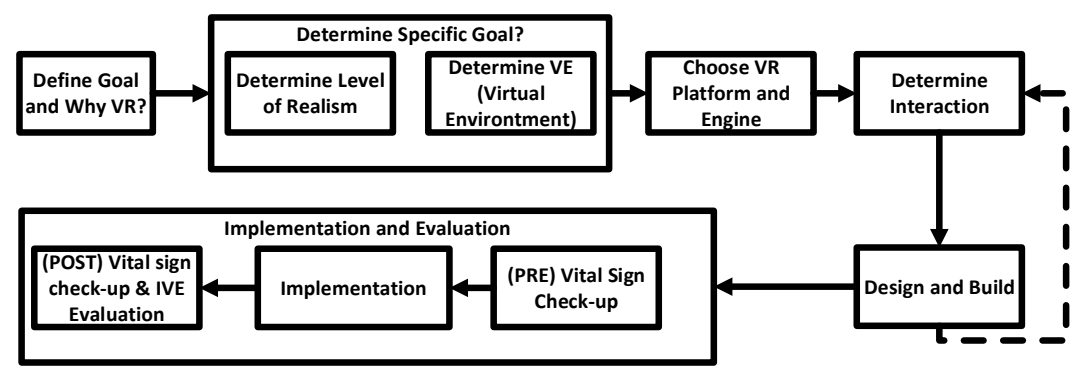

Fig. 1. The VR research methodology for exercise during pregnancy 
Step 1. Define the purpose and reason why using VR.

Step 2. Determine the more specific objectives, which divided into two sub-steps.

Step 2.1. Determine the level of realism. The level of realism measured on a scale from very symbolic to very real.

Step 2.2. Determine the type of immersion and presence required. The types of immersion are no immersive, partial immersive, and fully immersive. The immersive environment, enabling users to be able to feel the direct presence while in the virtual world. Presence measured on a scale from a low sense of presence to a strong presence.

Step 3. Select the VR (hardware) platform used and the VR engine.

Step 4. Determine the type of interaction with the input and output devices are related to the existing virtual environment.

Step 5. Design the virtual environments, the whole system, and build the system on a predefined VR platform.

Step 6. In this step, there are three steps of implementation and evaluation.

Step 6.1. Doing vital sign check-up to the target user before practice with VR.

Step 6.2. VR application implementation for the target user.

Step 6.3. Doing vital sign check-up after practice with VR and evaluation of IVE questionnaire [7].

\subsection{Define the purpose and reason why using VR}

In general, this research aims to develop VR application for pregnant women to easily perform an exercise during pregnancy with no limited space and time. The reason why the VR-based application selected because it allows pregnant women to see virtual environments that contain virtual prenatal personal trainer. So that pregnant women can easily learn or even practice exercises that have been taught by the virtual prenatal personal trainer.

\subsection{Define the purpose and reason why using VR}

The object of the study is on pregnant women with gestational age starting from the age of 16-18 weeks or the second trimester will be given treatment using pregnancy exercise application. Gestational age at weeks 16 to 18 , is a period in which the vital organs of the fetus is already formed and begins to form the extremities and maturation of vital organs, so exercise during pregnancy would not harm the fetus [22].

\subsection{Determine the level of realism, type of immersion, and presence}

In this VR app for pregnancy exercise, it uses a moderate level of realism, which means that the virtual environment that the expectant woman sees is not so symbolic and not so real. The type of immersion is fully immersive. So that pregnant women would be fully involved with the virtual entities and has a strong presence while in the virtual environment. Mobile VR selected as a platform to run this application, so it 
needs a smartphone and a VR headset. Mobile VR can be more easy to arrange and relatively inexpensive when compared to VR Desktop (HTC Vive and Oculus Rift). There is no cable plugged on Mobile VR, which can reduce the risk of falling pregnant women when using VR application.

\subsection{Determine the type of interaction}

VR mobile app development requires a smartphone, which has an inertial tracking sensor. Such inertial tracking sensors are accelerometer and gyroscope [23]. Google VR and Unity have been used to develop this app. Google VR developed by Google Developers that provides the virtual reality SDK for some development environment. The Google VR has several API's functions that can use for rendering, managing input, and enabling VR controller device. Unity is a game engine and editor to develop multimedia and games applications. However, it could be used to develop VR applications.

\subsection{Design and Build}

Fig. 2 depicts the complete system design. The communication between the user (pregnant women) and the VR system obtained from the VR headset and smartphone device. These devices act as input or output media. Continuous recurrence between the user and the virtual environment occurs until the user wants to end the received virtual experience.

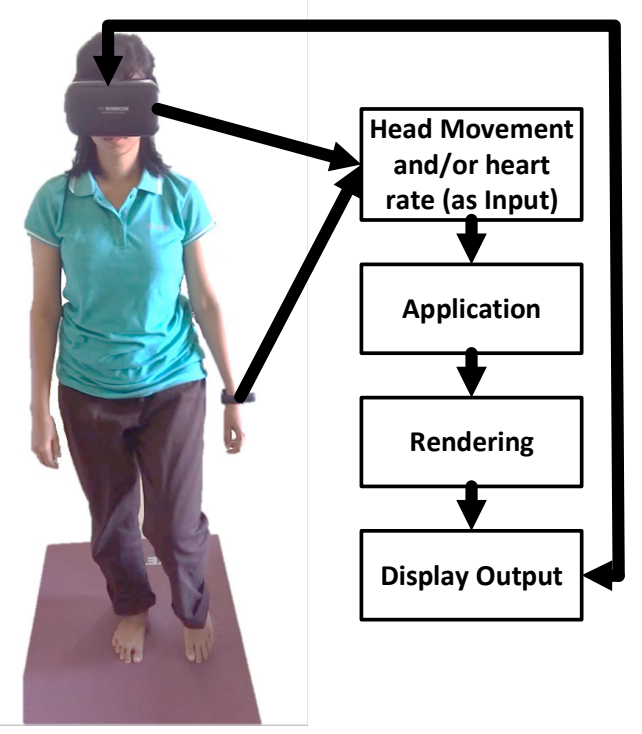

Fig. 2. The complete system design of VR for exercise during pregnancy 
The acceptable input interactions of the system are nodding moves that will be responded by the virtual prenatal personal trainer. The application receives input from the user and updates non-rendering aspects such as physics simulations and user interactions. Rendering is a transformation process that gives the illusion of reality to the user including visual rendering and auditory rendering on virtual trainer objects. The output is a physical representation received directly by the user through the display of smartphones and audio (headphones).

A nod of the head which is responded by the virtual trainer is conducted by ray casting the center of gaze to dummy object. Ray casting is a checking method using a ray of a particular point to look at the closest object that blocks the ray. Fig. 3 is a procedure of a simple nod movement algorithm, which implemented in the VR system for this pregnancy exercise.

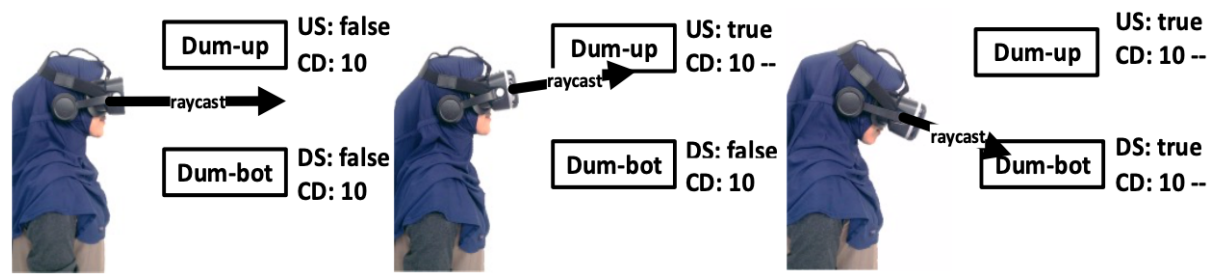

Fig. 3. The procedure of simple nod movement using VR system

For the initialization stage, a countdown timer (CD) variable starts in 10 seconds, an up state and downstate variable with the value of false. After initializing those variables, calculate the ray from the camera position (from the user's gaze position) with the constrained value of the maximum ray. Check whether the ray makes any collision with dummy object. When the dummy object is "Dum-up" or "Dum-bot" making collision with the ray, the countdown timer decrease from 10 to zero. While the value of countdown timer is not zero, the up state (US) or downstate (DS) variable will be true. The up state or downstate variable will be false if the countdown timer is zero. The user should move the head down immediately after seeing up to make a nodding movement accepted by the system.

In Unity SDK, performing the ray casting method was done by checking the call of the Raycast function. The Raycast parameter needs the reference of camera position transformation, the forward vector from the camera, and the length of the ray. The code snippet for checking of the ray collided with an object described below: 


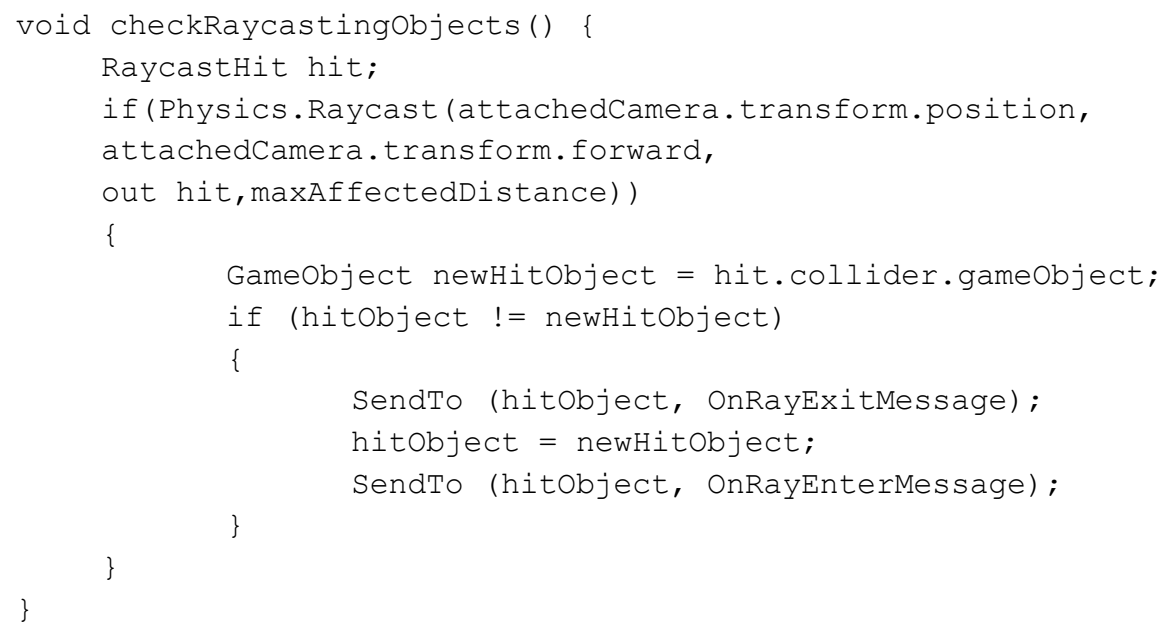

A RaycastHit object is an object which it returned to the collided object exposed by the ray. The event-listener mechanism started when the ray hits an object in the OnRayEnterMessage event-handler function. When ray has not hit an object then used OnRayExitMessage event-handler function.

Besides the ray casting mechanism to nod, there is a heart rate monitoring system on wearable device combined with VR system. The heart rate system is quite important considering its use for pregnant women. The system is used as an early warning system when the medical condition of pregnant women is unstable when using VR. It is sometimes not realized by pregnant women because it is too involved with the virtual environment. The wearable device used is Samsung Gear 2 that works on the Tizen operating system. Unlike Unity programming that uses C\# programming language, the Tizen uses Javascript as a programming language. To retrieve heart rate data on Tizen application system, the programmer used the privilege of Tizen.humanactivitymonitor API. Below the use of the API function in the following code implementation:

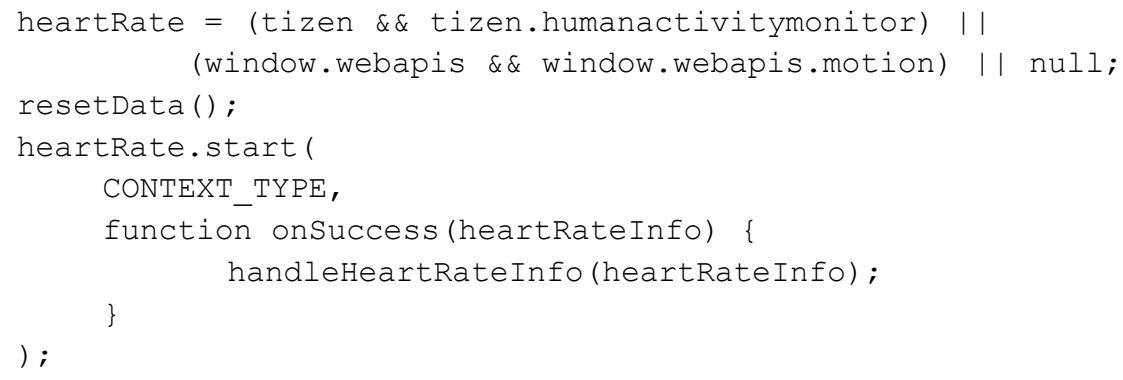

It needs a communication network to transmit data from the heart rate sensor of wearable device with the smartphone. The communication network which is used to transmit data is the internet connectivity. Both wearable device and smartphone must 
be connected to the internet to perform the data exchange. The data transmission mechanism for both wearable device and smartphone uses URL Request method. In the wearable device, the URL Request method performed to transmit heart rate data to the host server is as follows:

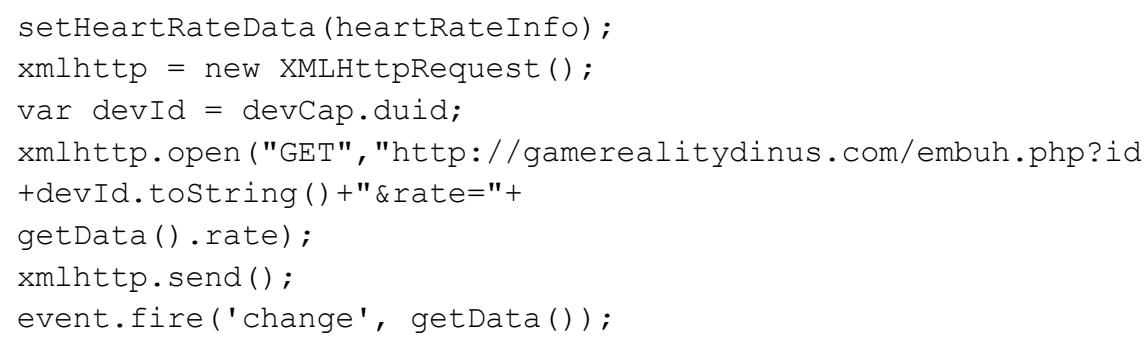

It requires the device identity checking to ensure that the wearable device used by the correct user. So it needs to take the Unique Identifier (UDID) of the wearable device. UDID and Heart rate data sent via internet connectivity. The data will be stored temporarily on the database server. In smartphone device, also use the Request URL mechanism to receive heart rate data from the database server. Previously, the user was asked to enter the UDID of wearable device manually. The UDID information appears in the first time opening screen of the heart rate monitor application in the wearable device. For every single heart rate, data will be recorded in every five seconds and will also store in the device's local storage.

After the interaction design and build finished, the next stage is virtual environment models creation. The major virtual environment that must exist in this VR system is the virtual prenatal personal trainer. The virtual prenatal trainer should be able to have some animation of pregnancy exercise movement and be able to interact with the user. The animation of virtual prenatal trainer movements has been predetermined, namely: the warm-up exercises, the main pregnancy exercises, and the warm-down exercises. The warm-up movement consists of hand and foot stretching like a regular exercise. Then proceed with the core activities of sitting straight leg forward, supine attitude and move the legs and ankles, then lift the hips/pelvis, and try to manage the breath. After that, sit cross-legged and stretch back and manage the breath that serves to prepare for normal childbirth. Some of the movements chosen in this study have been through consideration of the security and convenience aspects of the user while using VR devices.

Total sessions of all pregnancy exercise activities are ten sessions with two sessions of warm-up exercise, seven sessions of pregnancy exercise, and one session warm-down exercise. First, warm-up sessions performed with moving the hands and feet in a standing position. Second, the main pregnancy exercise sessions performed by sitting position with a stretching activity of leg and hand. Third, the warm-down session is carried out with the sitting condition by straightening the body by lifting both hands upward while managing the breath.

The additional ability of the virtual prenatal trainer is making any interaction with the user through the dialogue system generated by the object. The user needs to nod 
the head to respond the virtual prenatal trainer while making interaction and then the virtual prenatal trainer will continue the session. Other virtual environments are additional and visible to the user (with no interaction). The virtual environments are the field, the grass, and the rocks.

Once all the virtual environment components are available, the last is combining all the virtual environment with existing interactions to form the whole system. The internal testers and programmers will check VR system functionality. When there are no meaningful bugs, then the system will be built on Android platform and then can be implemented.

\section{Implementation and Evaluation}

The VR application of pregnancy exercise installed in Xiaomi Redmi Note 4 smartphone and we used VR Shinecon as VR headset. After the application evaluation finished by testers and programmers, the next stage is implementing the system to the pregnant woman. In the first time use, pregnant women asked for their availability for testing this application. Pregnant women will also be tested in advance about the medical condition of vital signs before starting this VR application. It is necessary considering the medical circumstances of pregnant women are different with normal women in general. Checking of vital signs is done by measuring heart rate (HR), blood pressure $(\mathrm{BD})$, respiration rate $(\mathrm{RR})$, and anemic conjunctiva $(\mathrm{AC})$. The testers will ask pregnant women if they have vomiting, nausea, and dizziness during pregnancy before using VR.

There are six pregnant women are willing to participate in the implementation of exercise during pregnancy with this VR application. Pregnant women are in the age range from 25 to 32 years with gestational age from 11 to 32 weeks. Table 1 shows demographic data and participant's vital data before performing the exercise with VR.

Table 1. Demographic and pre-implementation data of six pregnant women.

\begin{tabular}{|l|l|l|l|l|l|l|l|l|l|l|l|l|}
\hline $\begin{array}{c}\text { Par. } \\
\text { No. }\end{array}$ & $\begin{array}{c}\text { Ges.Age } \\
(\text { week) }\end{array}$ & \multirow{2}{*}{ Age } & \multirow{2}{*}{$\begin{array}{c}\text { Weight } \\
(\mathbf{k g})\end{array}$} & \multirow{2}{*}{$\begin{array}{c}\text { Height } \\
(\mathbf{m})\end{array}$} & \multirow{2}{*}{ VR? } & \multicolumn{6}{|c|}{ Pre Implementation } \\
\hline 1 & 26 & 27 & 57 & 1.56 & No & $85 / 65$ & 80 & 18 & yes & yes & yes & no \\
\hline 2 & 16 & 27 & 65 & 1.55 & No & $110 / 90$ & 80 & 20 & no & no & yes & no \\
\hline 3 & 11 & 27 & 53 & 1.58 & No & $90 / 60$ & 72 & 16 & yes & yes & yes & no \\
\hline 4 & 32 & 32 & 70 & 1.48 & No & $120 / 90$ & 100 & 18 & yes & no & no & no \\
\hline 5 & 23 & 28 & 65 & 1.58 & No & $110 / 70$ & 80 & 20 & yes & no & no & no \\
\hline 6 & 22 & 25 & 46 & 1.54 & No & $120 / 80$ & 80 & 18 & yes & yes & yes & no \\
\hline
\end{tabular}

After checking the vital signs, pregnant women will ask about the experience of using VR. Nobody has ever used VR for both ordinary apps and games. In the absence of VR experience experienced by pregnant women, the testers explain a brief explanation of how to use VR and its preparation. When the pregnant woman is ready to wear VR headset properly and comfortably, then pregnancy exercise can be started. Fig. 4 shows how pregnant women practices exercise during pregnancy with guided by a virtual prenatal personal trainer on the VR system. 


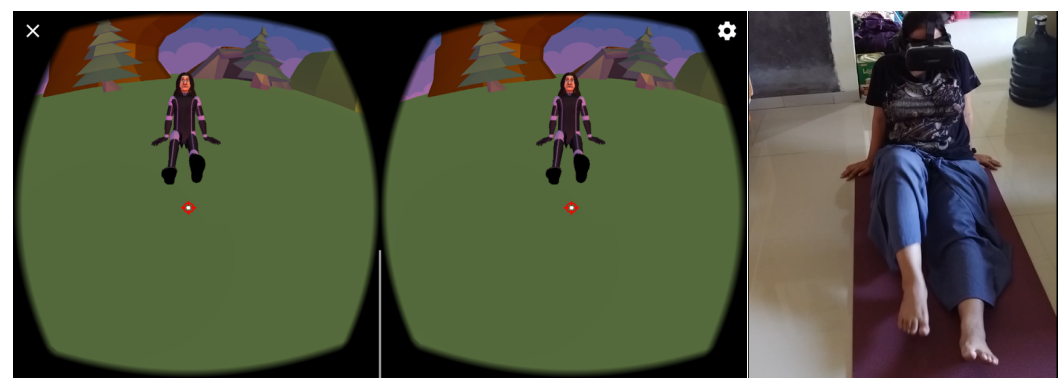

Fig. 4. Pregnant women practice exercise during pregnancy with guides of virtual prenatal personal trainer on the VR system

The final evaluation is to check the vital signs of pregnant women again and use the IVE questionnaire which given to a pregnant woman after they complete the exercise, to evaluate the VR system. The results of checking the vital signs after doing exercise with VR showed no sign of bad health condition. Table 2 shows the results of checking vital signs shortly after pregnant women doing exercise with VR. Besides, researchers can also see the heart rate recorded on the local storage of the smartphone device when pregnant women doing exercise with VR as seen in Fig. 5. The heart rate of a pregnant woman changes during exercise. After all pregnancy exercise sessions finished, the heart rate gradually returns to its initial condition.

Table 2. Post-implementation data of six pregnant women.

\begin{tabular}{|c|c|c|c|c|c|c|c|}
\hline \multirow{2}{*}{$\begin{array}{l}\text { Par. } \\
\text { No. }\end{array}$} & \multicolumn{7}{|c|}{ Post Implementation } \\
\hline & $B D$ & $H R$ & $R R$ & $A C$ & Vom. & Nau. & Diz. \\
\hline 1 & $90 / 70$ & 100 & 22 & yes & no & no & no \\
\hline 2 & $120 / 80$ & 100 & 24 & no & no & no & no \\
\hline 3 & $100 / 70$ & 80 & 20 & yes & no & no & no \\
\hline 4 & $110 / 80$ & 100 & 22 & no & no & no & no \\
\hline 5 & $120 / 80$ & 88 & 24 & yes & no & no & no \\
\hline 6 & $125 / 80$ & 100 & 22 & yes & no & no & no \\
\hline
\end{tabular}

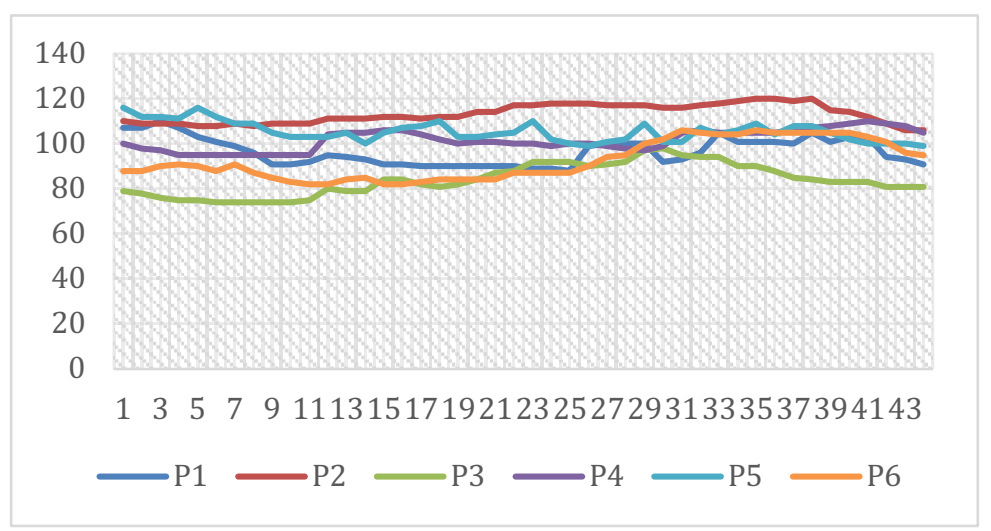

Fig. 5. The heart rate monitored while pregnant women practice exercise during pregnancy with VR system 
The next evaluation is testing with IVE questionnaire. The IVE questionnaire for this VR application for pregnancy exercise consists of 33 statements. The answer of participants are in the range of strongly agree, agree, neutral, disagree, and strongly disagree. For a positive statement, five-point scales numbered presented from 5 (anchored with "Strongly agree") to 1 (anchored with "Strongly disagree"). In contrast to the negative statement, the value of 5 is given from the strongly disagree to the value of 1 for strongly agree. A negative statement applied to all statement items of experience consequence. The other is a positive statement. For each component of the questionnaire item described in the list below:

- Presence: There are eight statements related to this component (e.g. "There are eight statements related to this component." "The virtual trainer can respond to my nodding movement correctly." "The movements of virtual trainer seemed natural.").

- Engagement: Only one statement on this component (e.g. "I felt excited when using the VR for pregnancy exercise.").

- Immersive: There are two statements related to this component (e.g. "I think that all virtual objects seemed real." "I became so involved in VR for pregnancy exercise that I lose all track of time.").

- Flow: Only one statement on this component (e.g. "I was not worried while exercise by using the VR for pregnancy exercise.").

- Usability: There are three statements related to this component (e.g. "The VR system is easy to use." "I think that the animation steps of a virtual trainer are working properly.").

- Skill: Only one statement on this component (e.g. "I feel confident understanding all functionality of the use of VR without no experience before.").

- Emotion: There are three statements related to this component (e.g. "I felt confident using a smartphone (not for the VR for pregnancy exercise)." "I enjoyed the new experience of pregnancy exercise with VR.").

- Judgment: Only one statement on this component (e.g. "I feel not nervous when trying doing pregnancy exercise with VR.").

- Technology Adoption: There are four statements related to this component (e.g. "I knew how to do pregnancy exercise after using the VR."."This system can make it easier for me to do pregnancy exercises whenever and wherever.").

- Experience Consequence: There are nine statements related to this component (e.g. "I suffered from nausea during exercise with the virtual environment.,,"I suffered from vertigo during exercise with the virtual environment.").

Fig. 6 depicts the results of the IVE questionnaire on all aspects of the IVE components performed in pregnant women. The lowest average result is on the aspect of the Judgment $(\mathrm{Ju})$ component with 3.67. The highest average yield on aspects of Skill (Sk) and Emotion (Em) components with 4.50. Nevertheless, the average of all aspects of the IVE questionnaire given is 4.26. From the evaluation of checking the vital signs and IVE questionnaire gave positive results for later application of VR pregnancy exercise is feasible to use. 


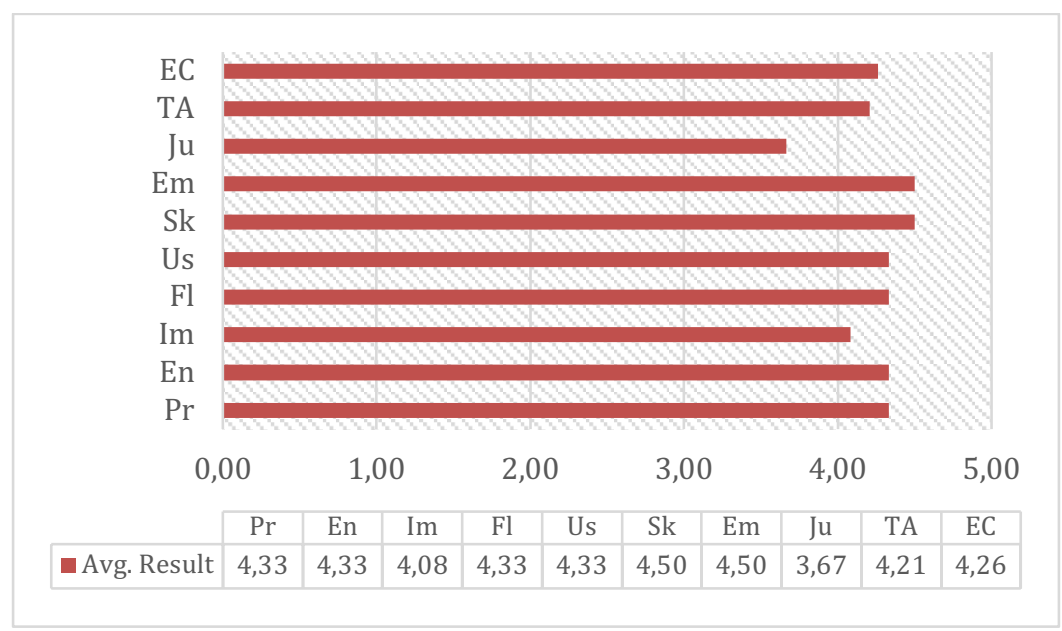

Fig. 6. The results of the IVE questionnaire for all aspects of the IVE components

\section{Conclusion}

VR technology has potentially used for health training especially for exercise during pregnancy. The existence of increasingly sophisticated smartphone device and the number of VR headsets sold freely can facilitate pregnant women to do exercise through this VR application for pregnancy exercise. Besides, pregnant women can also see the monitored heart rate while doing the exercises in a virtual environment using the wearable device. The methodology used to develop a VR application for pregnancy exercise is a modification of the methodology to determine when to use VR with the added evaluation method of the IVE questionnaire.

The average results of all aspects of the components in the IVE questionnaire showed positive results. That indicates the VR application for pregnancy exercise is feasible to use by pregnant women. In the future research, it is possible to develop Mixed Reality applications for pregnancy exercise so that when doing exercise, pregnant women still can see the environment in the real world. The multiple connections of several devices sensors are possible ways to make the VR or MR application to be more comfortable, interactive, and safe when used to a pregnant woman.

\section{Reference}

[1] United Nations Population Funds, "Maternal health," 2015. [Online]. Available:https://www.unfpa.org/maternal-health. [Accessed: 23-May-2018].

[2] C. Ward-Ritacco, M. S. Poudevigne, and P. J. O'Connor, "Muscle strengthening exercises during pregnancy are associated with increased energy and reduced fatigue," J. Psychosom. Obstet. Gynecol., vol. 37, no. 2, pp. 68-72, Apr. 2016. https://doi.org/10.3109/0167482X.2016.1155552 
[3] S. T. Harris, J. Liu, S. Wilcox, R. Moran, and A. Gallagher, "Exercise During Pregnancy and its Association with Gestational Weight Gain," Matern. Child Health J., vol. 19, no. 3, pp. 528-537, Mar. 2015. https://doi.org/10.1007/s10995-014-1534-8

[4] R. Barakat et al., "Exercise during pregnancy protects against hypertension and macrosomia: randomized clinical trial," Am. J. Obstet. Gynecol., vol. 214, no. 5, p. 649.e1649.e8, May 2016. https://doi.org/10.1016/j.ajog.2015.11.039

[5] C. Donalek et al., "Immersive and collaborative data visualization using virtual reality platforms," in 2014 IEEE International Conference on Big Data (Big Data), 2014, pp. 609614. https://doi.org/10.1109/BigData.2014.7004282

[6] V. S. Pantelidis, "Reasons to Use Virtual Reality in Education and Training Courses and a Model to Determine When to Use Virtual Reality," Themes Sci. Technol. Educ., vol. 2, pp. 59-70, 2009.

[7] K. Tcha-Tokey, E. Loup-Escande, O. Christmann, and S. Richir, "Proposition and Validation of a Questionnaire to Measure the User Experience," Int. J. Virtual Real., vol. 16, pp. 33-48, 2016.

[8] C. Xia, "Multimedia Teaching Platform Construction Based on Flash Interaction Technology for Gymnastics,” Int. J. Emerg. Technol. Learn., vol. 13, no. 5, p. 224, Apr. 2018. https://doi.org/10.3991/ijet.v13i05.8441

[9] S. De Ribaupierre, B. Kapralos, F. Haji, E. Stroulia, A. Dubrowski, and R. Eagleson, "Healthcare Training Enhancement Through Virtual Reality and Serious Games," in Virtual, Augmented Reality and Serious Games for Healthcare 1, Intelligent Systems Reference Library, vol. 68, Springer-Verlag Berlin Heidelberg 2014, 2014, pp. 9-28.

[10] Y. M. Kim, M. H. Chun, G. J. Yun, Y. J. Song, and H. E. Young, "The Effect of Virtual Reality Training on Unilateral Spatial Neglect in Stroke Patients," Ann. Rehabil. Med., vol. 35, no. 3, p. 309, 2011. https://doi.org/10.5535/arm.2011.35.3.309

[11] A. Mostafa, W. Ryu, S. Chan, K. Takashima, and G. Kopp, "Designing NeuroSimVR: A Stereoscopic Virtual Reality Spine Surgery Simulator,” Science (80-.), pp. 1-20, 2017.

[12] Y. Pulijala, M. Ma, M. Pears, D. Peebles, and A. Ayoub, "Effectiveness of Immersive Virtual Reality in Surgical Training-A Randomized Control Trial," J. Oral Maxillofac. Surg., vol. 76, no. 5, pp. 1065-1072, May 2018. https://doi.org/10.1016/j.jo ms.2017.10.002

[13] K. B. Chen et al., "Use of Virtual Reality Feedback for Patients with Chronic Neck Pain and Kinesiophobia," IEEE Trans. Neural Syst. Rehabil. Eng., vol. 25, no. 8, pp. 12401248, Aug. 2017. https://doi.org/10.1109/TNSRE.2016.2621886

[14] R. S. Monteiro-Junior et al., "Virtual Reality-Based Physical Exercise With Exergames (PhysEx) Improves Mental and Physical Health of Institutionalized O lder Adults," J. Am. Med. Dir. Assoc., vol. 18, no. 5, p. 454.e1-454.e9, May 2017. https://doi.org/10.1016/j.jamda.2017.01.001

[15] B. G. Witmer and M. J. Singer, "Measuring Presence in Virtual Environments: A Presence Questionnaire," Presence Teleoperators Virtual Environ. vol. 7, no. 3, pp. 225-240, Jun. 1998. https://doi.org/10.1162/105474698565686

[16] J. Heutte, "La part du collectif dans la motivation et son impact sur le bien-être comme médiateur de la réussite des étudiants : complémentarités et contributions entre l'autodétermination, l'auto-efficacité et l'autotélisme," Université de Nanterre - Paris X, 2011.

[17] J. R. Lewis and J. Sauro, "The Factor Structure of the System Usability Scale," vol. 1, 2009, pp. 94-103.

[18] C. A. Murphy, D. Coover, and S. V. Owen, "Development and Validation of the Computer Self-Efficacy Scale,” Educ. Psychol. Meas., vol. 49, no. 4, pp. 893-899, Dec. 1989. https://doi.org/10.1177/001316448904900412 
[19] R. Pekrun, T. Goetz, A. C. Frenzel, P. Barchfeld, and R. P. Perry, "Measuring emotions in students' learning and performance: The Achievement Emotions Questionnaire (AEQ)," Contemp. Educ. Psychol., vol. 36, no. 1, pp. 36-48, Jan. 2011. https://doi.org/10.1016/j.cedpsych.2010.10.002

[20] R. S. Kennedy, N. E. Lane, K. S. Berbaum, and M. G. Lilienthal, "Simulator Sickness Questionnaire: An Enhanced Method for Quantifying Simulator Sickness," Int. J. Aviat. Psychol., vol. 3, no. 3, pp. 203-220, Jul. 1993. https://doi.org/10.1207/s15327108ijap0303 3

[21] V. Venkatesh, J. Y. L. Thong, B. Statistics, X. Xu, and T. Acceptance, "Unified Theory of Acceptance and Use of Technology: A Synthesis and the Road Ahead," J. Assoc. Inf. Syst., vol. 17, no. 5, pp. 328-376, 2016. https://doi.org/10.17705/1jais.00428

[22] D. Masrurin, S. Subiyatun, and N. I. Rahmawati, "Minat Ibu Hamil dalam Mengikuti Senam Hamil di BPRB Bina Sehat Bangunjiwo Kasihan, Bantul," J. Ners Midwifery Indones., vol. 1, no. 1, pp. 12-17, 2013. https://doi.org/10.21927/jnki.2013.1(1).12-17

[23] W. Fang, L. Zheng, H. Deng, and H. Zhang, "Real-Time Motion Tracking for Mobile Augmented/Virtual Reality Using Adaptive Visual-Inertial Fusion," Sensors, vol. 17, no. 5, p. 1037, May 2017. https://doi.org/10.3390/s17051037

\section{Acknowledgment}

This research supported by Penelitian Dosen Pemula (PDP) grant of the Directorate of Research and Community Service, the Directorate General for Research and Development of the Ministry of Research, Technology and Higher Education of the Republic of Indonesia.

Abas Setiawan is a lecturer in Computer Science Faculty, Dian NuswantoroUniversity, Semarang, Jawa Tengah, Indonesia

Faik Agiwahyuanto is a lecturer in Health Science Faculty, Dian Nuswantoro University, Semarang, Jawa Tengah, Indonesia.

Pramudi Arsiwi is a lecturer in Engineering Faculty, Dian Nuswantoro University, Semarang, Jawa Tengah, Indonesia.

Article submitted 31 May 2018. Resubmitted 15 July 2018. Final acceptance 03 August 2018. Final version published as submitted by the author. 